\title{
Regionally Specific Human GABA Concentration Correlates with Tactile Discrimination Thresholds
}

\author{
Nicolaas A. J. Puts, ${ }^{1,2}$ Richard A. E. Edden, ${ }^{3,4}$ C. John Evans, ${ }^{1}$ Francis McGlone, ${ }^{5}$ and David J. McGonigle ${ }^{1,2}$ \\ ${ }^{1}$ Cardiff University Brain Research Imaging Centre, School of Psychology, Cardiff University, CF10 3AT Cardiff, United Kingdom, ${ }^{2}$ School of Biosciences, \\ Cardiff University, CF10 3AX Cardiff, United Kingdom, ${ }^{3}$ Russell H. Morgan Department of Radiology and Radiological Science, The Johns Hopkins \\ University School of Medicine, Baltimore, Maryland, ${ }^{4}$ F. M. Kirby Center for Functional Brain Imaging, Kennedy Krieger Institute, Baltimore, Maryland, \\ and ${ }^{5}$ Faculty of Science, Liverpool John Moores University, L3 3AF Liverpool, United Kingdom
}

The neural mechanisms underlying variability in human sensory perception remain incompletely understood. In particular, few studies have attempted to investigate the relationship between in vivo measurements of neurochemistry and individuals' behavioral performance. Our previous work found a relationship between GABA concentration in the visual cortex and orientation discrimination thresholds (Edden et al., 2009). In the present study, we used magnetic resonance spectroscopy of GABA and psychophysical testing of vibrotactile frequency thresholds to investigate whether individual differences in tactile frequency discrimination performance are correlated with GABA concentration in sensorimotor cortex. Behaviorally, individuals showed a wide range of discrimination thresholds ranging from 3 to $7.6 \mathrm{~Hz}$ around the $25 \mathrm{~Hz}$ standard. These frequency discrimination thresholds were significantly correlated with GABA concentration $(r=-0.58 ; p<0.05)$ in individuals' sensorimotor cortex, but not with GABA concentration in an occipital control region $(r=-0.04)$. These results demonstrate a link between GABA concentration and frequency discrimination in vivo, and support the hypothesis that GABAergic mechanisms have an important role to play in sensory discrimination.

\section{Introduction}

GABAergic inhibition plays an important role in defining the selectivity of cortical responses to behaviorally relevant stimuli. The application of GABAergic antagonists, such as bicuculline, has been shown to broaden the responses of individual neurons to visual (Sillito, 1975; Tsumoto et al., 1979; Sillito et al., 1980; Wolf et al., 1986), auditory (Müller and Scheich, 1988; Fuzessery and Hall, 1996) and somatosensory (Dykes et al., 1984; Alloway and Burton, 1986; Juliano et al., 1989) stimuli.

We have recently shown that an alternative strategy to pharmacological manipulation for investigating the relationship between GABAergic inhibition and behavior is to compare individual differences in GABA concentration in vivo to psychophysical measurements (Edden et al., 2009; Boy et al., 2010; Sumner et al., 2010). For example, in the visual system, we have shown that orientation discrimination thresholds correlate with GABA concentration in visual cortex (Edden et al., 2009), in the sense that those participants who perform well at the orientation discrimination task have more GABA in their occipital regions.

\footnotetext{
Received Sept. 2, 2011; accepted Sept. 22, 2011.

Author contributions: N.A.J.P., R.A.E.E., and D.J.M. designed research; N.A.J.P., C.J.E., and D.J.M. performed research; F.M. contributed unpublished reagents/analytic tools; N.A.J.P., R.A.E.E., C.J.E., and D.J.M. analyzed data; N.A.J.P., R.A.E.E., C.J.E., F.M., and D.J.M. wrote the paper.

This work was supported by the Schools of Psychology and Biosciences at Cardiff University, and by the Welsh Institute of Cognitive Neuroscience. R.A.E.E. held a Research Councils UK (RCUK) Academic Fellowship. D.J.M. holds an RCUK Academic Fellowship.

The authors declare no competing financial interests.

Correspondence should be addressed to Dr. David J. McGonigle, Tower Building, School of Psychology, Cardiff University, Park Place, Cardiff CF10 3AT, UK. E-mail: mcgonigled@cardiff.ac.uk.

DOI:10.1523/JNEUROSCI.4489-11.2011

Copyright $\odot 2011$ the authors $\quad 0270-6474 / 11 / 3116556-05 \$ 15.00 / 0$
}

In the somatosensory system, vibrotactile frequency discrimination in the flutter range $(5-40 \mathrm{~Hz})$ is regularly used as a behavioral paradigm to investigate the neuronal correlates of sensory encoding and decision making (Mountcastle et al., 1967, 1969, 1997; LaMotte and Mountcastle, 1975; Hernández et al., 2000b; Salinas et al., 2000; Romo et al., 2002). While much is currently known about how representations of vibration frequency are gradually transformed into motor outputs by successive cortical regions (Romo and Salinas, 2003; Luna et al., 2005; Hernández et al., 2010), the nature of interindividual differences on this task is less clear. Although the excessive amounts of training required and small cohort sizes make it difficult to investigate individual differences in nonhuman primates, learning studies have shown that increases in individual tactile performance through training can be linked directly to changes in neuronal responses in primary somatosensory cortex (S1; Recanzone et al., 1992). As GABAergic mechanisms have been previously suggested as a basis for these functional alterations, here we directly test the hypothesis that differences in GABA between individuals can account for differences in perceptual performance. We used magnetic resonance spectroscopy (MRS), which is currently the only noninvasive methodology that can measure the endogenous concentration of neurotransmitters and metabolites, to measure GABA concentrations in cortical regions. We further tested the hypothesis that the relationship between brain GABA concentration and behavioral performance is regionally specific by comparing GABA measurements in the sensorimotor region with similar measurements in an occipital (control) region. 


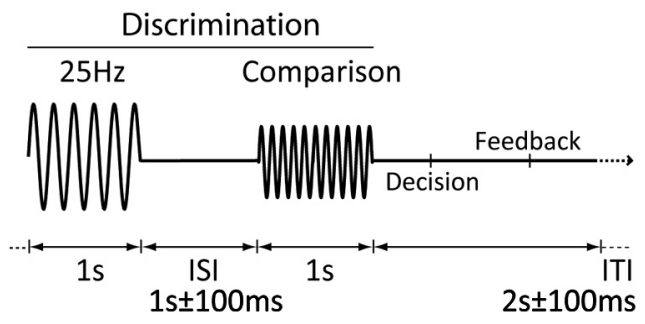

Figure 1. Frequency discrimination paradigm. Frequency discrimination thresholds to a 25 $\mathrm{Hz}$ stimulus were determined for frequencies higher and lower than $25 \mathrm{~Hz}$ in a $2 \mathrm{AFC}$ paradigm. The order of presentation of the standard $(25 \mathrm{~Hz})$ and comparison stimuli were randomized.
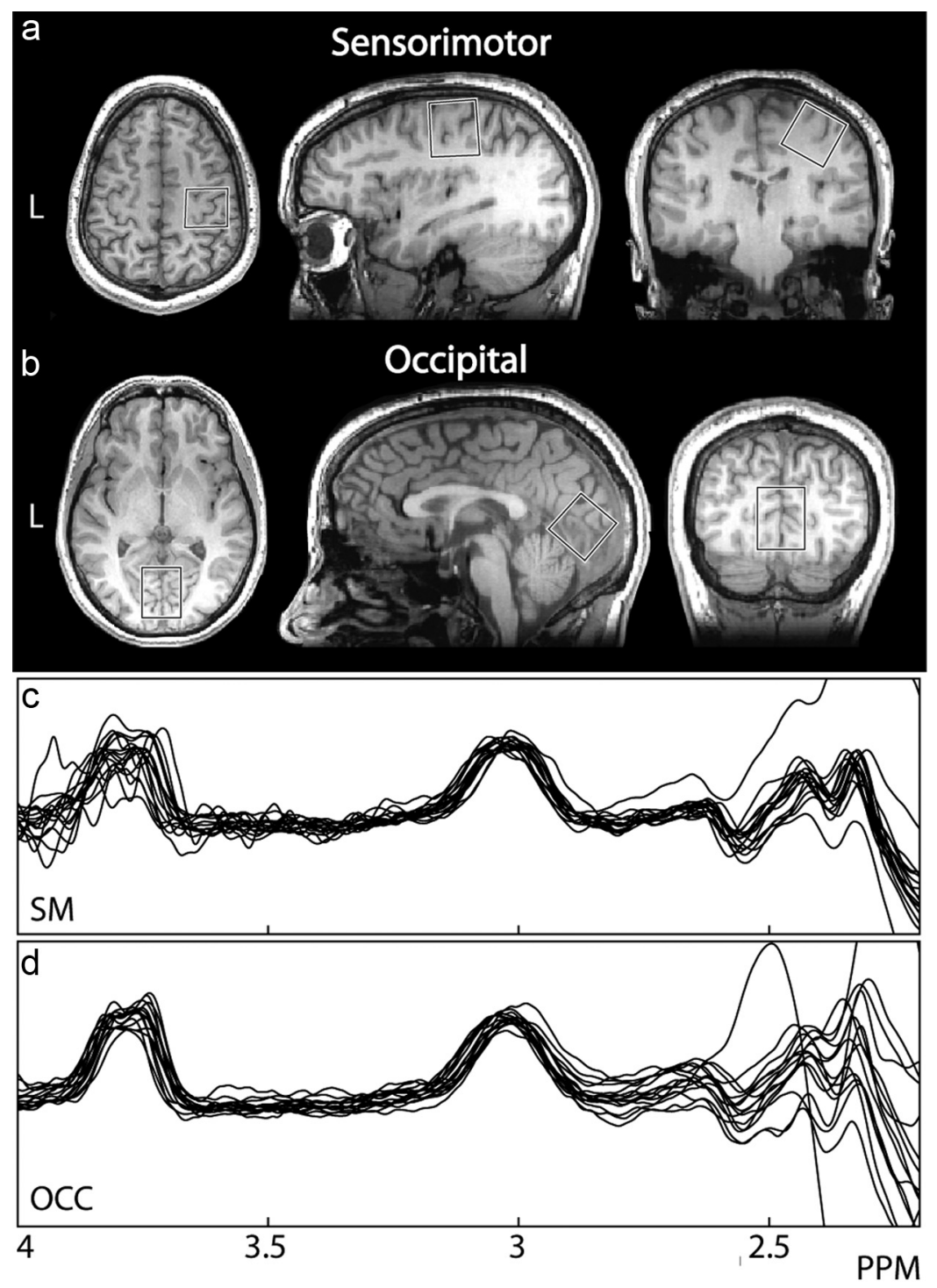

Figure 2. Voxel locations and spectra. $\boldsymbol{a}$, A single-participant example voxel over the right sensorimotor cortex. The center of the voxel is placed on the "hand knob," an anatomical landmark indicating the hand area of the primary motor cortex, with the hand area of primary somatosensory cortex, directly posterior across the central sulcus, also included (Yousry et al., 1997). $\boldsymbol{b}$, An example voxel over occipital cortex. $\boldsymbol{c}, \boldsymbol{d}$, High-quality spectra from all participants for the sensorimotor (SM) and occipital cortex (OCC) voxels respectively, with a clearly distinguishable GABA peak at 3 ppm for all participants.

\section{Materials and Methods}

Participants. Sixteen right-handed participants (mean age 27.3; SD 4.3; range $22-34 ; 10$ male) were recruited for this study. None had a history of neurological disease. All participants gave informed consent and all procedures were approved by the local ethics committee at Cardiff University's School of Psychology. One participant was excluded from further analysis due to poor execution of the behavioral task, which prevented the determination of threshold in this individual.

Behavioral psychophysics. Vibrotactile stimulation was delivered using a piezoelectric vibrotactile stimulator (McGlone et al., 2002). Contact with the skin was made via a plastic probe $(7 \mathrm{~mm}$ diameter). A static surround limited the stimulation to the skin region directly under the probe. All stimulation was delivered to the glabrous skin of left digit 2 (index finger). Stimuli were delivered via the audio output of a laptop computer (Sony Vaio VGN-NS20M, Realtek high-definition audio) using Matlab 2008b (The MathWorks, 2008). A $25 \mathrm{~Hz} 1 \mathrm{~s}$ sinusoid was used as the "target" for detection and as the "standard" for discrimination. The PEST algorithm (Taylor and Creelman, 1967) was used to determine all threshold measurements.

Participants were seated comfortably in a dedicated behavioral testing room at Cardiff University Brain Research Imaging Centre (CUBRIC) and their finger was placed on the stimulator. Before the frequency discrimination task, individual detection threshold values were determined using a 60-trial two-interval forced-choice [interval duration $1 \mathrm{~s} ; 1 \pm 100$ $\mathrm{ms}$ inter-stimulus interval (ISI)] paradigm in which the amplitude of the target was altered in a stepwise fashion to find participants' $75 \%$ correct threshold. The resulting amplitude was increased by $8 \mathrm{~dB}$ for the discrimination task (after LaMotte and Mountcastle, 1975). Vibrotactile discrimination thresholds around the standard were measured using a 100-trial two-track interleaved two-alternative forcedchoice design (2AFC; $1 \pm 100$ ms ISI) with stimulus frequency as the adaptive variable (Fig. 1). The order of the tracks was pseudorandomized, so that a maximum of five trials of the same track occurred sequentially. The order of standard and comparison stimuli was randomized between trials.

Frequency discrimination thresholds were calculated by taking the modulus average of the last 15 trials across the two tracks. For the task, intertrial intervals consisted of a $2 \mathrm{~s}$ pause with $\pm 100 \mathrm{~ms}$ jitter to focus attention and reduce expectation of stimulus occurrence. Previous frequency discrimination studies have shown that perceived intensity varies as a function of frequency as well as intensity (LaMotte and Mountcastle, 1975; Verrillo and Capraro, 1975), so the amplitude of the comparison stimulus was determined by a normalized subjective frequency-intensity matching curve.

MR experiment. After vibrotactile thresholds were determined, subjects proceeded to the MR scanning suite at CUBRIC. All scanning was performed on a GE SignaHDx 3 tesla MRI scanner (General Electric Healthcare), using an eight-element head coil for receive and the body coil for transmit. Before MRS acquisition, a $1 \mathrm{~mm}^{3}$ isotropic-resolution T1-weighted anatomical scan (FSPGR) was acquired to determine voxel placement. GABA-edited MR spectra were acquired using the MEGAPRESS method (Mescher et al., 1998; Edden and Barker, 2007) in two (3 $\mathrm{cm})^{3}$ volumes in the right sensorimotor and midline occipital regions (Fig. 2), while subjects were at rest in the MR scanner. Due to the experimental limitations of MRS, GABA measurements must be made from a 
volume including both primary somatosensory and motor cortex, which we designate "sensorimotor" cortex.

Sensorimotor and visual voxel localization was determined as described previously (Evans et al., 2010), except the sensorimotor voxel was rotated in both the sagittal and coronal planes so that one face of the voxel was parallel to the cortical surface. The following acquisition parameters were used: TR/TE $1800 / 68 \mathrm{~ms} ; 332$ scans of 2048 data points per $10 \mathrm{~min}$ acquisition; $16 \mathrm{~ms}$ Gaussian editing pulse applied at $1.9 \mathrm{ppm}(\mathrm{ON})$ and $7.46 \mathrm{ppm}(\mathrm{OFF})$ in interleaved scans.

GABA concentration was quantified in institutional units from the ratio of the edited GABA signal at $3 \mathrm{ppm}$ (as determined by fitting with a simple Gaussian model) to the unsuppressed water signal from the same volume. All processing of MRS data was performed using in-house software written in MATLAB. GABA concentrations were corrected for the voxel tissue fraction (white matter + gray matter) obtained using FAST (Zhang et al., 2001). FreeSurfer was used to obtain cortical thickness measurements of the precentral and postcentral gyri, with each gyrus defined by an automated parcellation technique (Dale et al., 1999; Fischl and Dale, 2000; Fischl et al., 2004). Estimates of cortical thickness and gray matter volume were also computed for the part of each gyrus that was contained within our sensorimotor MRS voxel for each subject.

Statistical analysis. All results are quoted as mean \pm SD. Correlations between GABA concentration, frequency discrimination threshold, and structural anatomical parameters were tested using the Pearson correlation coefficients $(r)$. Group differences between male and female participants were tested using Student's $t$ test.

\section{Results}

Average frequency discrimination thresholds to the $25 \mathrm{~Hz}$ standard were $4.9 \pm 1.23 \mathrm{~Hz}$. These values are consistent with previous studies measuring frequency discrimination thresholds in healthy adults to stimuli in the flutter range (Goble and Hollins, 1994; Tommerdahl et al., 2005).

High-quality MRS spectra were obtained in all participants (as shown in Fig. 2), showing a clear edited signal at 3 ppm. GABA concentration in sensorimotor cortex was found to correlate significantly $(r=-0.58, p<0.05)$ with frequency discrimination threshold. Participants with a higher level of GABA performed better at the task, as shown in Figure 3. To test the effects of outliers on our correlations, we performed a jackknife analysis: the data were resampled $n$ times ( $n=$ number of subjects), excluding one subject each time, and the correlation was computed. For the correlation between GABA concentration and discrimination threshold, this produced a range of $r$ values between -0.53 and -0.68 , all of which were significant at $p<0.05$. No correlation was seen between GABA concentration in occipital cortex and discrimination threshold $(r=-0.04 ; p>0.5$; jackknifed $r$ range -0.2175 to 0.1123 , all of which were NS).

As studies have suggested links between macroanatomical structural features of the sensorimotor cortical area and aspects of motor and sensory abilities in individuals (e.g., Gaser and Schlaug, 2003), we measured the cortical thickness and total gray matter volume of both precentral and postcentral gyri in each subject. In addition, we calculated the cortical thickness and gray matter volume of those areas of either gyri that were contained within our MRS voxel, allowing us to separate the anatomy of the voxel into primarily motor (precentral) and tactile (postcentral) regions. No significant correlations were found between any of these measures of anatomy and either tactile discrimination thresholds or GABA concentration in the sensorimotor voxel.

No significant correlation was seen between GABA concentration and age within this relatively homogeneous cohort $(r=$ $-0.08)$. There was no significant difference in concentration between male and female participants for either region $(p>0.3$ for both regions).

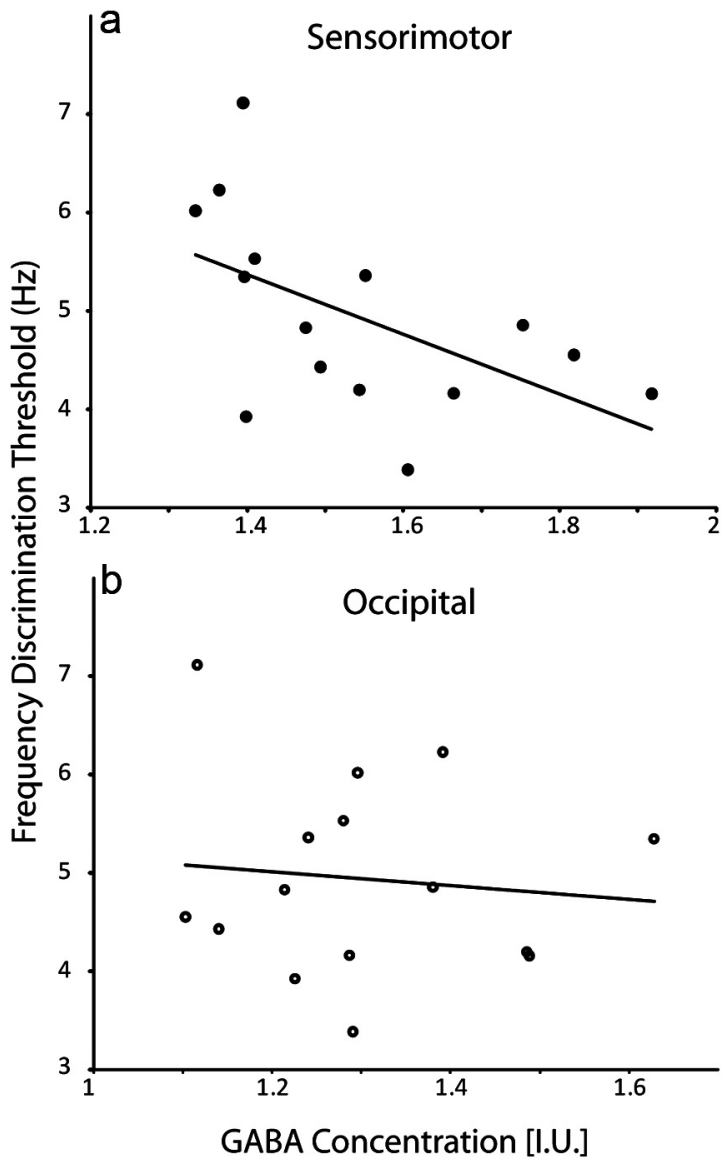

Figure 3. $\boldsymbol{a}$, Individual GABA concentration in sensorimotor cortex correlates significantly with frequency discrimination threshold $(r=-0.58, p<0.05)$. $\boldsymbol{b}, \mathrm{GABA}$ concentration in occipital cortex does not correlate with tactile frequency discrimination threshold ( $r=-0.04$, NS). GABA concentrations are corrected for tissue volume (gray matter + white matter).

\section{Discussion}

Our findings show a significant correlation between GABA concentration and tactile frequency discrimination threshold only in the sensorimotor cortex voxel. Higher GABA concentrations in this region predict lower discrimination thresholds, consistent with our previous study linking orientation discrimination and GABA in the visual cortex (Edden et al., 2009).

\section{GABAergic influences on tactile discrimination}

How might differences in GABA concentration, measured at the coarse spatial scale of our MRS data, determine individual differences in tactile function? The mechanisms linking GABAergic inhibition to tactile processing are incompletely appreciated at the cellular level, and while this study has focused on the mechanism by which individual differences in tactile sensitivity are reflected by individual GABA levels, our results do not provide further insights into how frequency discrimination is encoded in human participants with no previous experience on the task. However, they do suggest that the GABAergic system plays a crucial role in determining individual differences in frequency discrimination in healthy adults.

Studies have shown that GABAergic inhibition acts via the lateral inhibition of neighboring neurons or columns in somatosensory cortex to influence spatial receptive fields in SI (Lee and Whitsel, 1992). This adaptive spatial ability may also be paralleled by a similar mechanism in the temporal domain. Fast-spiking 
(FS) GABAergic interneurons exert powerful effects on the precision of spike timing in pyramidal cells (Bacci and Huguenard, 2006), and recent work (Cardin et al., 2009) has demonstrated a causal link between oscillations in the gamma frequency range $(20-80 \mathrm{~Hz}), \mathrm{FS}$ interneuron activity, and the efficacy of sensory processing. Neurons in area $3 \mathrm{~b}$ explicitly encode information about flutter frequency, firing in phase with each stimulus cycle of the mechanical vibrations (Mountcastle et al., 1969; Hernández et al., 2000a). Perceptual learning studies (Recanzone et al., 1992; Wang et al., 1995), have suggested that improvements in tactile discrimination may be driven by a "sharpening" in the tuning of neuronal responses to the indentation cycles of vibrotactile stimuli. This would enhance the signal-to-noise ratio (SNR) of the sensory response, and in doing so improve the fidelity of the stimulus representation (as suggested in Harris et al., 2001). Similarly, reductions in the efficacy of GABAergic transmission are known to reduce the SNR of tactile frequency encoding in SI (McLaughlin and Juliano, 2005), and age-related changes in the SNR of visual cortical neurons are affected by GABA levels (Leventhal et al., 2003). If there exists a temporal "window of opportunity" (Knoblich et al., 2010) to ensure the encoding success or failure of a given sensory stimulus by a given cortical region (first suggested by Mountcastle et al., 1990), then it is likely that inhibitory GABAergic mechanisms play an important role in its generation and maintenance.

\section{MRS measurements of baseline GABA concentration}

We do not think that our results merely reflect a nonspecific increase in neuronal density or surface cortical thickness that could underlie the differences in perceptual ability between subjects. However, as previous work has suggested links between gross anatomical structure and behavior in the cortical regions within our sensorimotor voxel, we performed a number of control analyses, exploring whether the variability in either GABA concentration or tactile discrimination thresholds could be explained by cortical thickness or gray matter volume in the precentral and postcentral gyri. None of these analyses were significant, suggesting that the correlation between GABA and behavior in our data is unlikely to be driven by gross anatomical differences between subjects.

Rather, as suggested by histological measurements in nonhuman primates (Hendry et al., 1987), our measurements may instead reflect the proportion of GABAergic inhibitory interneurons in a given cortical area. Due to the ubiquity of GABA's involvement in signal transmission in cortex and its complex, reciprocal relationship with excitatory transmission, we propose here that our measurements of baseline GABA concentration provide a way to sample the efficacy of normal cortical function in a given brain region.

In addition, our study shows that GABA concentration in occipital cortex, a sensory region that is not thought to play a role in tactile frequency encoding, does not correlate with the frequency discrimination thresholds. This further supports previous work (Boy et al., 2010; Sumner et al., 2010) that GABA concentration, as measured by MRS, varies in a behaviorally relevant manner across the brain.

\section{GABAergic function and sensory processing in neuropsychiatric disorders}

Our findings have significance for several neuropsychiatric disorders in which a link between alterations in sensory processing and GABAergic mechanisms is hypothesized, most notably autistic spectrum disorder (Tannan et al., 2008; Tommerdahl et al.,
2008) and schizophrenia (Chang and Lenzenweger, 2005). Both these disorders are heterogeneous in presentation and are likely to be caused by a range of factors, rather than a deficit to a single neurotransmitter system. While pharmacologic intervention has been used to address comorbid features of autism, such as anxiety, hyperactivity, and aggressive behavior, there are no currently available medications that effectively target the core social/communicative and behavioral features of autism, including sensory processing. It is possible that GABAergic interventions may represent a future goal for drug development in these disorders.

\section{Limits of MEGA-PRESS measurements of GABA}

The MEGA-PRESS technique used to measure GABA still has several limitations. The size of the voxel used is relatively large $\left(3 \times 3 \times 3 \mathrm{~cm}^{3}\right)$ and it is therefore not possible to place a voxel specifically on the primary somatosensory cortex. Although other studies of GABA have used smaller volumes (for review, see Puts and Edden, 2011), correlative studies of individual differences require optimal signal-to-noise, and the reliance on PRESS localization gives a cuboidal excitation volume that is fundamentally different from the curved geometry of S1. However, we believe that the link between somatosensory function and concentrations of GABA that we describe here is most parsimoniously explained by considering that the individual differences on the discrimination task are primarily driven by individual differences in the GABA signal from S1. At 3T, the MEGA-PRESS methodology results in significant excitation of coedited macromolecule signal, which contributes $\sim 40 \%$ of the edited signal at 3 ppm. We believe that that the behavioral effects observed are more easily explained by GABA than by the macromolecular signal.

\section{Conclusions}

In conclusion, the present study is the first to link somatosensory discrimination performance to GABAergic inhibition by using the observable range among healthy individuals. Combined with previous studies in visual cortex, this suggests a general role for GABAergic inhibition in behavioral discrimination in healthy participants.

\section{References}

Alloway KD, Burton H (1986) Bicuculline-induced alterations in neuronal responses to controlled tactile stimuli in the second somatosensory cortex of the cat: a microiontophoretic study. Somatosens Res 3:197-211.

Bacci A, Huguenard JR (2006) Enhancement of spike-timing precision by autaptic transmission in neocortical inhibitory interneurons. Neuron 49:119-130.

Boy F, Evans CJ, Edden RA, Singh KD, Husain M, Sumner P (2010) Individual differences in subconscious motor control predicted by GABA concentration in SMA. Curr Biol 20:1779-1785.

Cardin JA, Carlén M, Meletis K, Knoblich U, Zhang F, Deisseroth K, Tsai LH, Moore CI (2009) Driving fast-spiking cells induces gamma rhythm and controls sensory responses. Nature 459:663-667.

Chang BP, Lenzenweger MF (2005) Somatosensory processing and schizophrenia liability: proprioception, exteroceptive sensitivity, and graphesthesia performance in the biological relatives of schizophrenia patients. J Abnorm Psychol 114:85-95.

Dale AM, Fischl B, Sereno MI (1999) Cortical surface-based analysis. I. Segmentation and surface reconstruction. Neuroimage 9:179-194.

Dykes RW, Landry P, Metherate R, Hicks TP (1984) Functional role of GABA in cat primary somatosensory cortex: shaping receptive fields of cortical neurons. J Neurophysiol 52:1066-1093.

Edden RA, Barker PB (2007) Spatial effects in the detection of gammaaminobutyric acid: improved sensitivity at high fields using inner volume saturation. Magn Reson Med 58:1276-1282.

Edden RA, Muthukumaraswamy SD, Freeman TC, Singh KD (2009) Orientation discrimination performance is predicted by GABA concentration 
and gamma oscillation frequency in human primary visual cortex. J Neurosci 29:15721-15726.

Evans CJ, McGonigle DJ, Edden RA (2010) Diurnal stability of gammaaminobutyric acid concentration in visual and sensorimotor cortex. J Magn Reson Imaging 31:204-209.

Fischl B, Dale AM (2000) Measuring the thickness of the human cerebral cortex from magnetic resonance images. Proc Natl Acad Sci U S A 97:11050-11055.

Fischl B, van der Kouwe A, Destrieux C, Halgren E, Ségonne F, Salat DH, Busa E, Seidman LJ, Goldstein J, Kennedy D, Caviness V, Makris N, Rosen B, Dale AM (2004) Automatically parcellating the human cerebral cortex. Cereb Cortex 14:11-22.

Fuzessery ZM, Hall JC (1996) Role of GABA in shaping frequency tuning and creating FM sweep selectivity in the inferior colliculus. J Neurophysiol 76:1059-1073.

Gaser C, Schlaug G (2003) Brain structures differ between musicians and non-musicians. J Neurosci 23:9240-9245.

Goble AK, Hollins M (1994) Vibrotactile adaptation enhances frequency discrimination. J Acoust Soc Am 96:771-780.

Harris JA, Harris IM, Diamond ME (2001) The topography of tactile learning in humans. J Neurosci 21:1056-1061.

Hendry SH, Schwark HD, Jones EG, Yan J (1987) Numbers and proportions of GABA-immunoreactive neurons in different areas of monkey cerebral cortex. J Neurosci 7:1503-1519.

Hernández A, Zainos A, Romo R (2000a) Neuronal correlates of sensory discrimination in the somatosensory cortex. Proc Natl Acad Sci U S A 97:6191-6196.

Hernández A, Zainos A, Romo R (2000b) Neuronal correlates of sensory discrimination in the somatosensory cortex. Proc Natl Acad Sci U S A 97:6191-6196.

Hernández A, Nácher V, Luna R, Zainos A, Lemus L, Alvarez M, Vázquez Y, Camarillo L, Romo R (2010) Decoding a perceptual decision process across cortex. Neuron 66:300-314.

Juliano SL, Whitsel BL, Tommerdahl M, Cheema SS (1989) Determinants of patchy metabolic labeling in the somatosensory cortex of cats: a possible role for intrinsic inhibitory circuitry. J Neurosci 9:1-12.

Knoblich U, Siegle JH, Pritchett DL, Moore CI (2010) What do we gain from gamma? Local dynamic gain modulation drives enhanced efficacy and efficiency of signal transmission. Front Hum Neurosci 4:185.

LaMotte RH, Mountcastle VB (1975) Capacities of humans and monkeys to discriminate vibratory stimuli of different frequency and amplitude: a correlation between neural events and psychological measurements. J Neurophysiol 38:539-559.

Lee CJ, Whitsel BL (1992) Mechanisms underlying somatosensory cortical dynamics: I. In vivo studies. Cereb Cortex 2:81-106.

Leventhal AG, Wang Y, Pu M, Zhou Y, Ma Y (2003) GABA and its agonists improved visual cortical function in senescent monkeys. Science 300:812-815.

Luna R, Hernández A, Brody CD, Romo R (2005) Neural codes for perceptual discrimination in primary somatosensory cortex. Nat Neurosci 8:1210-1219.

McGlone F, Kelly EF, Trulsson M, Francis ST, Westling G, Bowtell R (2002) Functional neuroimaging studies of human somatosensory cortex. Behav Brain Res 135:147-158.

McLaughlin DF, Juliano SL (2005) Disruption of layer 4 development alters laminar processing in ferret somatosensory cortex. Cereb Cortex 15:1791-1803.

Mescher M, Merkle H, Kirsch J, Garwood M, Gruetter R (1998) Simultaneous in vivo spectral editing and water suppression. NMR Biomed 11:266-272.

Mountcastle VB, Talbot WH, Darian-Smith I, Kornhuber HH (1967) Neural basis of the sense of flutter-vibration. Science 155:597-600.
Mountcastle VB, Talbot WH, Sakata H, Hyvärinen J (1969) Cortical neuronal mechanisms in flutter-vibration studied in unanesthetized monkeys. Neuronal periodicity and frequency discrimination. J Neurophysiol 32:452-484.

Mountcastle VB, Steinmetz MA, Romo R (1990) Frequency discrimination in the sense of flutter: psychophysical measurements correlated with postcentral events in behaving monkeys. J Neurosci 10:3032-3044.

Müller CM, Scheich H (1988) Contribution of GABAergic inhibition to the response characteristics of auditory units in the avian forebrain. J Neurophysiol 59:1673-1689.

Puts NAJ, Edden RAE (2011) In vivo magnetic resonance spectroscopy of GABA: a methodological review. Prog NMR Spect. Advance online publication. doi:10.1016/j.pnmrs.2011.06.001.

Recanzone GH, Jenkins WM, Hradek GT, Merzenich MM (1992) Progressive improvement in discriminative abilities in adult owl monkeys performing a tactile frequency discrimination task. J Neurophysiol 67:1015-1030.

Romo R, Salinas E (2003) Flutter discrimination: neural codes, perception, memory and decision making. Nat Rev Neurosci 4:203-218.

Romo R, Hernández A, Zainos A, Lemus L, Brody CD (2002) Neuronal correlates of decision-making in secondary somatosensory cortex. Nat Neurosci 5:1217-1225.

Salinas E, Hernandez A, Zainos A, Romo R (2000) Periodicity and firing rate as candidate neural codes for the frequency of vibrotactile stimuli. J Neurosci 20:5503-5515.

Sillito AM (1975) The contribution of inhibitory mechanisms to the receptive field properties of neurones in the striate cortex of the cat. J Physiol 250:305-329.

Sillito AM, Kemp JA, Milson JA, Berardi N (1980) A re-evaluation of the mechanisms underlying simple cell orientation selectivity. Brain Res 194:517-520.

Sumner P, Edden RA, Bompas A, Evans CJ, Singh KD (2010) More GABA, less distraction: a neurochemical predictor of motor decision speed. Nat Neurosci 13:825-827.

Tannan V, Holden JK, Zhang Z, Baranek GT, Tommerdahl MA (2008) Perceptual metrics of individuals with autism provide evidence for disinhibition. Autism Res 1:223-230.

Taylor MM, Creelman CD (1967) PEST: efficient estimates and probability functions. J Acoust Soc Am 41:782-787.

Tommerdahl M, Hester KD, Felix ER, Hollins M, Favorov OV, Quibrera PM, Whitsel BL (2005) Human vibrotactile frequency discriminative capacity after adaptation to $25 \mathrm{~Hz}$ or $200 \mathrm{~Hz}$ stimulation. Brain Res 1057:1-9.

Tommerdahl M, Tannan V, Holden JK, Baranek GT (2008) Absence of stimulus-driven synchronization effects on sensory perception in autism: evidence for local underconnectivity? Behav Brain Funct 4:19.

Tsumoto T, Eckart W, Creutzfeldt OD (1979) Modification of orientation sensitivity of cat visual cortex neurons by removal of GABA-mediated inhibition. Exp Brain Res 34:351-363.

Verrillo RT, Capraro AJ (1975) Effect of stimulus frequency on subjective vibrotactile magnitude functions. Atten Percept Psychophys 17:91-96.

Wang X, Merzenich MM, Sameshima K, Jenkins WM (1995) Remodelling of hand representation in adult cortex determined by timing of tactile stimulation. Nature 378:71-75.

Wolf W, Hicks TP, Albus K (1986) The contribution of GABA-mediated inhibitory mechanisms to visual response properties of neurons in the kitten's striate cortex. J Neurosci 6:2779-2795.

Yousry TA, Schmid UD, Alkadhi H, Schmidt D, Peraud A, Buettner A, Winkler P (1997) Localization of the motor hand area to a knob on the precentral gyrus. A new landmark. Brain 120:141-157.

Zhang Y, Brady M, Smith S (2001) Segmentation of brain MR images through a hidden Markov random field model and the expectationmaximization algorithm. IEEE Trans Med Imaging 20:45-57. 